\title{
INTEGRATING IMPERFECTION OF INFORMATION INTO THE PROMETHEE MULTICRITERIA DECISION AID METHODS: A GENERAL FRAMEWORK
}

\author{
Sarah BEN AMOR*, Bertrand MARESCHAL**
}

\begin{abstract}
Multicriteria decision aid methods are used to analyze decision problems including a series of alternative decisions evaluated on several criteria. They most often assume that perfect information is available with respect to the evaluation of the alternative decisions. However, in practice, imprecision, uncertainty or indetermination are often present at least for some criteria. This is a limit of most multicriteria methods. In particular the PROMETHEE methods do not allow directly for taking into account this kind of imperfection of information. We show how a general framework can be adapted to PROMETHEE and can be used in order to integrate different imperfect information models such as a.o. probabilities, fuzzy logic or possibility theory. An important characteristic of the proposed approach is that it makes it possible to use different models for different criteria in the same decision problem.
\end{abstract}

Keywords: multiple criteria analysis, multicriteria decision aid, imperfection of information, PROMETHEE

\section{Introduction}

We consider discrete multicriteria decision problems where $A=\left\{a_{1}, a_{2}, \ldots, a_{n}\right\}$ is a set of $n$ alternative decisions (actions) that are evaluated on a set of $k$ criteria denoted by $f_{1}, f_{2}, . ., f_{k}$. The actions represent either potential decisions among which a choice has to be made or items to evaluate. The criteria express the different and often conflicting objectives of the decision-maker. Without loss of generality, we may assume that these criteria are realvalued and have to be maximized. The multicriteria decision problem can thus be written as follows:

* Telfer School of Management, University of Ottawa, Ottawa, Canada. (benamor@telfer.uottawa.ca)

** Solvay Brussels School of Economics and Management, Université Libre de Bruxelles, Brussels, Belgium (bmaresc@ulb.ac.be). 


$$
\max \left\{f_{1}(a), f_{2}(a), \ldots, f_{k}(a) \mid a \in A\right\}
$$

Where we note the evaluation of action $a_{i}$ on criterion $f_{j}$ as follows:

$$
e_{i j}=f_{j}\left(a_{i}\right)
$$

Different approaches have been proposed to treat multicriteria problems. They are based either on the absolute evaluation of the alternatives (such as multi-attribute utility (MAUT) or value functions methods) or either on pair-wise comparisons (a.o. outranking methods). The interested reader can for instance consult [6] for additional information.

In this paper we focus on the PROMETHEE and GAIA outranking methods ([1], [3], [4]). According to [1] and to the online bibliographical database available at http://biblio.promethee-gaia.net, these methods are amongst the most widely used in practice. They have also been implemented in several recognized software such as PROMCALC, Decision Lab and more recently the PROMETHEE Software. With respect to other outranking methods, such as ELECTRE ([6]), PROMETHEE is based on a relatively simple principle that makes it easier to extend for taking into account imperfection of information. It also includes the GAIA descriptive approach.

PROMETHEE is a prescriptive method that enables to rank the actions according to the preferences of the decision-maker. Actually two rankings are produced: a partial ranking is built mostly on undisputable preferences and a complete, possibly less robust, ranking can also be obtained depending on the decision-maker's requirements. GAIA is a descriptive method. It complements PROMETHEE by providing the decision-maker with a synthetic visual representation of the main characteristics of the decision problem, such as the conflicts existing between the criteria and the specific profiles of the actions. GAIA can also play a role in the determination of the decision-maker priorities as the weights of the criteria and their impact on the PROMETHEE rankings are displayed.

As with most multicriteria decision aid methods PROMETHEE assume that perfect information is available for all the actions and all the criteria. That means that as well the evaluations of the actions on the criteria as the various preference parameters are known as exact numbers.

In practice, this is not always the case. There can be missing evaluations or imprecise information. We only consider here the case of the evaluations. For different reasons, this information can be difficult to obtain: there can be uncertainty on the future outcome of decisions, there can be some imprecision according to the evaluation procedure, or there can be indetermination associated to the measurement scales. Some attempts have been made to take this into account in the PROMETHEE methods. For instance missing values are integrated into the Decision Lab software. A stochastic extension of the PROMETHEE methods has also been proposed in [8] but it was limited to stochastic modeling and has not been implemented.

In section II we propose a general framework for handling different types of imperfection of information. We consider different modeling languages that can be used jointly in this context. Indeed several methods rely on probability theory to model the imperfection of information, some others rely on fuzzy logic, but very few allow the use of different modeling languages in the same framework [2]. In fact, various models are available such as possibility theory, fuzzy logic, interval arithmetic or the theory of evidence. They differ by their underlying assumptions and by the kind of information they 
require from the decision maker. The proposed framework makes it possible to use the best suited model for each criterion in a given decision problem and to thus combine all the models as needed.

In section III we recall the basic principles underlying the PROMETHEE \& GAIA methods. A more complete description of these methods, including a complete numerical example, can be found in [4].

In section IV we show how the general framework of section II can be integrated in PROMETHEE.

Section V concludes the paper. It summarizes the possibilities of the proposed approach and outlines future researches.

\section{A general framework for handling imperfection of information}

We consider only the imperfection of information appearing in the evaluations $e_{i j}$.

The general framework we use accepts, in the uncertain decision-making situations, stochastic, possibilistic or "evidential" evaluations. It also includes fuzzy or ordinal evaluations. Among all uncertainty-modeling languages (evidence theory, possibility theory and probability theory), the evidence theory presents the more general framework where possibilities and probabilities are proposed as particular cases. This property is used to settle the modeling framework.

Let us consider imperfect information for criterion $f_{j}$. For each alternative $a_{i}$, the evaluation $e_{i j}$ of $a_{i}$ on $f_{j}$ will depend on the actual state of the nature within a set of $H$ possible nature states:

$$
\Omega^{j}=\left\{\omega_{1}^{j}, \ldots, \omega_{h}^{j}, \ldots, \omega_{H}^{j}\right\}
$$

We note $x_{i j}^{h}$ the value of $e_{i j}$ under nature state $\omega_{h}^{j}$ and

$$
X_{i j}=\left\{x_{i j}^{1}, \ldots, x_{i j}^{h}, \ldots, x_{i j}^{H}\right\}
$$

This model assumes that we are able to indicate the consequence of choosing an alternative $a_{i}$ when the state of the nature $\omega_{j}^{h}(h=1, \ldots, H)$ occurs, i.e. when $x_{i j}^{h}$ is obtained.

The a priori information available about the states of the nature (probability functions, belief masses, possibility measures, ...) can be integrated as in Table 1.

This table is based on a theory of evidence model settings [12], where a priori information is represented by belief masses associated to focal elements. The focal elements, which are subsets of the states of the nature set:

$$
B_{h^{\prime}}^{j} \subseteq \Omega^{j}=\left\{\omega_{1}^{j}, \ldots, \omega_{h}^{j}, \ldots, \omega_{H}^{j}\right\} \quad h^{\prime}=1, \ldots H^{\prime} \leq 2^{H}
$$

determine the evaluations of the alternatives according to criterion $f_{j}$.

The evaluation $e_{i j}$ of action $a_{i}$ on $f_{j}$ is represented by a subset of values: 


$$
C_{i j}^{h^{\prime}} \subseteq X_{i j}
$$

which depends on the subsets of the states of the nature (focal elements) $B_{h^{\prime}}^{j}$.

Table 1. Performance matrix for criterion $f_{j}$ integrating the a priori information

\begin{tabular}{|c|c|c|c|c|c|c|}
\hline \multirow[t]{2}{*}{ Alternatives } & \multicolumn{5}{|c|}{ Focal elements } & \\
\hline & $B_{1}^{j}$ & $\cdots$ & $B_{h^{\prime}}^{j}$ & $\cdots$ & $B_{H^{\prime}}^{j}$ & \\
\hline$a_{1}$ & $C_{1 j}^{1}$ & $\cdots$ & $C_{1 j}^{h^{\prime}}$ & $\cdots$ & $C_{1 j}^{H^{\prime}}$ & \\
\hline$\vdots$ & $\vdots$ & & $\vdots$ & & $\vdots$ & \\
\hline$a_{i}$ & $C_{i j}^{1}$ & $\cdots$ & $C_{i j}^{h^{\prime}}$ & $\cdots$ & $C_{i j}^{H^{\prime}}$ & \\
\hline$\vdots$ & $\vdots$ & & $\vdots$ & & $\vdots$ & \\
\hline$a_{n}$ & $C_{n j}^{1}$ & $\cdots$ & $C_{n j}^{h^{\prime}}$ & $\cdots$ & $C_{n j}^{H^{\prime}}$ & \\
\hline $\begin{array}{c}\text { A priori belief } \\
\text { masses }\end{array}$ & $m\left(B_{1}^{j}\right)$ & & $m\left(B_{h^{\prime}}^{j}\right)$ & $\cdots$ & $m\left(B_{H \prime}^{j}\right)$ & $\cdots$ \\
\hline
\end{tabular}

By using this modeling framework, we can consider possibilistic or probabilistic models as particular cases when the a priori information is represented by possibility or probability distributions respectively. In fact, when the criterion is a possibilistic one, the a priori information related to the evaluation $e_{i j}$ is characterized by possibility distributions $\pi$. In such a context, the corresponding belief masses are associated with focal elements that are embedded:

$$
B_{1}^{j} \subseteq \ldots \subseteq B_{h^{\prime}}^{j} \subseteq \cdots \subseteq B_{H^{\prime}}^{j}
$$

The possibility measures coincide with the plausibilities of the embedded focal elements. When the criterion $f_{j}$ is stochastic, the focal elements $B_{h^{\prime}}^{j}$ are reduced to the singletons $\left\{\omega_{h}^{j}\right\}$ and the corresponding belief masses correspond to probability measures. In this case, the evaluations are characterized by random variables $X_{i j}^{h}$ with a priori probability distributions $f_{i j}^{h}$. We then have the a priori (subjective) probabilities $P_{j}^{h}$ for each state of the nature $\omega_{h}^{j}(h=1, \ldots, H)$.

The next step of our procedure consists of establishing the local preference relations between two actions. Let $H_{j}$ be the local relation between two actions $a_{i}$ and $a_{l}$ on criterion $f_{j}$. We have: $a_{i} H_{j} a_{l}$ where $H_{j} \in\left\{\succ ; \succ^{-1} ; \approx ; ?\right\}$ where $\approx$ is the indifference relation, $\succ$ is the strict preference relation, $\succ^{-1}$ is the inverse strict preference relation and ? is the incomparability relation.

In order to construct these relations, we privilege the approach based on the extension of the stochastic dominance concept. The use of the stochastic dominance concept has the advantage of providing a uniform treatment for the different languages which express the information imperfections. Notice that stochastic dominance allows to conclude about the preference of an action over another action for a decision-maker whose attitude towards 
risk corresponds to DARA (Decreasing Absolute Risk Aversion) utility functions [9]. This type of risk aversion is observed for several economic phenomena. The link between stochastic dominance and the preference is well known for this class of utility functions. It is not always easy to make the decision-maker's preferences explicit and we can often conclude that is preferred to if some stochastic dominance conditions are verified.

We consider the three first degrees of stochastic dominance. These are defined for continuous and discrete random variables in the following way. Let us note respectively $x_{j^{*}}$ and $x_{j}^{*}$ the lower and upper limits of the evaluation scale of criterion $f_{j} . F_{i j}$ and $F_{l j}$ are the cumulative probability distributions for the evaluations of alternatives $a_{i}$ and $a_{l}$ through criterion $f_{j}$ and $x_{j}$ is a modality of this criterion.

Definition 1: First order stochastic dominance (FSD)

$F_{i j} F S D F_{l j}$ if and only if

$$
H_{1}\left(x_{j}\right)=F_{i j}\left(x_{j}\right)-F_{l j}\left(x_{j}\right) \leq 0 \quad \forall x_{j} \in\left[x_{j^{*}}, x_{j}^{*}\right]
$$

Definition 2: Second order stochastic dominance (SSD)

$F_{i j} S S D F_{l j}$ if and only if

$$
H_{2}\left(x_{j}\right)=\int_{x_{j^{*}}}^{x_{j}} H_{1}(y) d y \leq 0 \quad \forall x_{j} \in\left[x_{j^{*}}, x_{j}^{*}\right]
$$

Definition 3: Third order stochastic dominance (TSD)

$F_{i j} T S D F_{l j}$ if and only if

$$
H_{3}\left(x_{j}\right)=\int_{x_{j^{*}}}^{x_{j}} H_{2}(y) d y \leq 0 \quad \forall x_{j} \in\left[x_{j^{*}}, x_{j}^{*}\right]
$$

In the case where the evaluation $e_{i j}$ is a random variable, the results of stochastic dominance can be directly applied in order to establish preference relations.

For instance, we could say that:

$$
e_{i j} \approx e_{l j} \Leftrightarrow\left|H_{1}\left(x_{j}\right)\right|=\left|F_{i j}\left(x_{j}\right)-F_{l j}\left(x_{j}\right)\right| \leq s_{j} \quad \forall x_{j} \in\left[x_{j^{*}}, x_{j}^{*}\right]
$$

where $s_{j}>0$ is a predetermined threshold.

Otherwise, for cases where $\left|H_{1}(x)\right|>s_{j},\left(F_{i j} \neq F_{l j}\right)$, we can say that:

$$
\begin{gathered}
e_{i j} \succ e_{l j} \Leftrightarrow F_{i j}(F S D \text { or } S S D \text { or } T S D) F_{l j} \\
e_{i j} ? e_{l j} \Leftrightarrow F_{i j} \operatorname{non} S D F_{l j}
\end{gathered}
$$

where $S D$ means that one of the three dominance types is verified. 
These concepts can be extended to ambiguous probabilities [7]. For fuzzy, possibilistic or evidential criteria, we propose the use of some transformations which provide to the functions characterizing the evaluation of an alternative on a given criterion (fuzzy membership functions, possibility distributions, belief masses, ...) properties similar to those of a probability density function.

For evidential criteria we use the pignistic transformation justified by Smets [13], [14]:

$$
\begin{aligned}
\operatorname{Bet} P\left(\omega_{h}^{j}\right) & =\sum_{B_{h^{\prime}}^{j} \omega_{h}^{j} \in B_{h^{\prime}}^{j}} \frac{m\left(B_{h^{\prime}}^{j}\right)}{\left|B_{h^{\prime}}^{j}\right|(1-m(\varnothing))} \\
\forall B_{h^{\prime}}^{j} & \in P(\Omega), m(\varnothing) \neq 1
\end{aligned}
$$

where $\left|B_{h^{\prime}}^{j}\right|$ is the cardinality of $\Omega$ in $B_{h^{\prime}}^{j}$ and $\operatorname{Bet} P\left(\omega_{h}\right)$ is the pignistic probability of $\omega_{h^{\prime}}$.

For possibilistic criteria we use the relation proposed by Dubois and al. [a]. This relation is equivalent to the pignistic transformation when applied to possibility measures. The probability $p_{h}$ corresponding to $\omega_{h}$ is given by:

$$
p_{h}=\sum_{t=h}^{H} \frac{1}{t} m_{t}=\sum_{t=h}^{H} \frac{1}{t}\left(\Pi_{t}-\Pi_{t+1}\right)
$$

where $m_{h}$ is the belief mass $m\left(B_{h}\right), B_{h}$ are embedded and organized in sequence, i.e. $B_{1}=\left\{\omega_{1}\right\}, B_{2}=\left\{\omega_{1}, \omega_{2}\right\}, \ldots, B_{h}=\left\{\omega_{1}, \omega_{2}, \ldots, \omega_{h}\right\}, \ldots, B_{H}=\Omega$ such that $\forall B \neq B_{h}$ : $m(B)=0$ and it is possible for a certain $h$ that $m\left(B_{h}\right)=0, \Pi_{h}=\Pi\left(\left\{\omega_{h}\right\}\right)$ and $\Pi$ is the possibility measure defined for $\left\{\omega_{h}\right\}$ :

$$
\Pi\left(\left\{\omega_{h}\right\}\right)=\sum_{t=h}^{H} m_{t}
$$

For fuzzy criteria we use the rescaling approach as proposed in Munda [10]:

$$
\begin{gathered}
f_{i j}\left(x_{i j}\right)=k_{i j} \mu_{i j}\left(e_{i j}\right) \\
\text { where } \int_{-\infty}^{\infty} f_{i j}\left(x_{i j}\right) d x_{i j}=1
\end{gathered}
$$

Let us note that the construction of local preference relations for deterministic criteria can be carried out by using discrimination thresholds to distinguish between strict preference and indifference situations (quasi-criterion notion) in the context of punctual evaluations. In this case, locally there is no incomparability. It is one of the characteristics of a criterion [11]. 


\section{The PROMETHEE Methods}

\subsection{Basic principles}

The PROMETHEE methods are based on a principle of pair-wise comparisons of the actions. We recall here the basic principles of the methods. A complete description can be found in [4] or on the PROMETHEE web site (http://www.promethee-gaia.net).

In a first step, a preference function has to be associated with each criterion in order to reflect the perception of the criterion scale by the decision-maker. Usually the preference function $P_{j}\left(a_{i}, a_{l}\right)$ is a non-decreasing function of the difference $f_{j}\left(a_{i}\right)-f_{j}\left(a_{l}\right)$ between the evaluations of two actions $a_{i}$ and $a_{l}$. Several typical shapes of preference functions are proposed in the literature $([1],[3])$ and indications are given on the way to select appropriate functions for different types of criteria. The value of $P_{j}\left(a_{i}, a_{l}\right)$ is a number between 0 and 1 . It corresponds to the degree of preference that the decision-maker expresses for $a_{i}$ over $a_{l}$ according to criterion $f_{j}$ : 0 corresponds to no preference at all while 1 corresponds to a full preference.

In a second step the decision-maker assesses numerical weights to the criteria to reflect the priorities: more important criteria receive larger weights. We note $w_{j}$ the weight of criterion $f_{j}$ and we assume that the weights are normalized as follows:

$$
\sum_{j=1}^{k} w_{j}=1
$$

A multicriteria pair-wise preference index is then computed as a weighted average of the preference functions:

$$
\pi\left(a_{i}, a_{l}\right)=\sum_{j=1}^{k} w_{j} P_{j}\left(a_{i}, a_{l}\right)
$$

Three preference flows are then computed in order to globally evaluate each action with respect to all the other ones.

The leaving flow is a measure of the strength of an action $a_{i}$ with respect to the other ones:

$$
\phi^{+}\left(a_{i}\right)=\frac{1}{n-1} \sum_{l=1}^{n} \pi\left(a_{i}, a_{l}\right)
$$

The entering flow measures the weakness of action $a_{i}$ with respect to the other ones:

$$
\phi^{-}\left(a_{i}\right)=\frac{1}{n-1} \sum_{l=1}^{n} \pi\left(a_{l}, a_{i}\right)
$$

Finally the net flow is the balance between the two first ones: 


$$
\begin{aligned}
\phi\left(a_{i}\right) & =\frac{1}{n-1} \sum_{l=1}^{n}\left(\pi\left(a_{i}, a_{l}\right)-\pi\left(a_{l}, a_{i}\right)\right) \\
& =\phi^{+}\left(a_{i}\right)-\phi^{-}\left(a_{i}\right)
\end{aligned}
$$

Each preference flow induces a ranking on the set of actions. Obviously the best actions should have a high $\phi^{+}$value (close to 1 ) and a low $\phi^{-}$value (close to 0 ), and thus a high positive $\phi$ value.

The PROMETHEE rankings are based on the preference flows. They are discussed in the next section.

The GAIA descriptive method is based on unicriterion net preference flows. These are computed in a similar way to (6) but for each criterion separately. For criterion $f_{j}$, the unicriterion net flow is defined as follows:

$$
\begin{aligned}
\phi_{j}\left(a_{i}\right) & =\frac{1}{n-1} \sum_{l=1}^{n}\left(P_{j}\left(a_{i}, a_{l}\right)-P_{j}\left(a_{l}, a_{i}\right)\right) \\
\phi\left(a_{i}\right) & =\sum_{j=1}^{k} w_{j} \phi_{j}\left(a_{i}\right)
\end{aligned}
$$

\subsection{PROMETHEE rankings}

The PROMETHEE I ranking is obtained by looking at the leaving (20) and entering (21) flows and keeping the preferences that are confirmed by both flows. It is a partial ranking as the two flows usually give a different ranking of the actions because they synthesize the pair-wise comparisons of the actions in two different ways. Their common part can thus be considered as more reliable.

The PROMETHEE II complete ranking is obtained by the net flow values (22). All actions are compared but the differences between the leaving and entering flows are lost, leading to a possibly less robust ranking.

\subsection{GAIA}

The PROMETHEE analysis is prescriptive. It relies on the preference parameters determined by the decision-maker. Changes in these parameters, especially the weights of the criteria, can have an important impact on the PROMETHEE rankings.

The GAIA analysis is based on the unicriterion net flows (23). Each action is then represented by a point in the $k$-dimensional space defined by these flows. A principal components analysis is then applied to these points to obtain a two-dimensional representation of the decision problem ([1], [6]). Unit axes for the criteria are also projected on the GAIA plane in order to show the conflicts between the criteria. 


\section{Imperfection of information in the PROMETHEE methods}

Introducing imperfection of information in the PROMETHEE methods can be done at different levels. Indeed the PROMETHEE methods rely not only on the evaluations of the actions on the criteria but also on preference modeling parameters such as the preference function thresholds and the weights of the criteria. For the latter, sensitivity analyses are one possible way to take into account the imperfection of information. Such analyses are made possible in the PROMETHEE software ([4]). We therefore focus on the evaluations.

As it is shown in section III, the PROMETHEE methods rely on pair-wise comparisons of actions. In case of perfect information, the evaluations are real numbers whose values are known precisely. The values of the preference functions $P_{j}\left(a_{i}, a_{l}\right)$ are then computed directly from the deviations between the evaluations. Let us note:

$$
p_{i l}^{j}=P_{j}\left(a_{i}, a_{l}\right)
$$

From the definition of the PROMETHEE preference functions, we have the following properties:

$$
0 \leq p_{i l}^{j} \leq 1 \text { and } p_{i l}^{j}+p_{l i}^{j} \leq 1
$$

Moreover both coefficients cannot be greater than 0 at the same time as in the deterministic case, preference is only possible in one way. As PROMETHEE uses valued preferences, we are limited to the situations in Table 2. In particular, no incomparability is possible in this case.

Table 2. PROMETHEE local preference relations in the deterministic case

\begin{tabular}{|c|c|c|}
\hline$p_{i l}^{j}$ & $p_{l i}^{j}$ & Local preference relation \\
\hline 0 & 0 & $\approx$ - Indifference \\
1 & 0 & $\succ$ - strict preference \\
$0<\delta<1$ & 0 & weaker preference \\
0 & 1 & $\succ^{-1}$ - inverse strict preference \\
0 & $0<\delta<1$ & inverse weaker preference \\
\hline
\end{tabular}

In case of imperfect information, an alternative way of computation of the $P_{j}\left(a_{i}, a_{l}\right)$ values must be defined. We propose to use the local preference relations as they are defined in Section III based on the general framework and on stochastic dominance. This approach corresponds to Table 3 where the PROMETHEE preference coefficients are determined according to the local preference relation.

Table 3. PROMETHEE preference coefficients in imperfect information case

\begin{tabular}{|c|c|c|}
\hline Local preference relation & $p_{i l}^{j}$ & $p_{l i}^{j}$ \\
\hline$\approx$ - Indifference & 0 & 0 \\
$\succ$ - strict preference & 1 & 0 \\
$\succ^{-1}$ - inverse strict preference & 0 & 1 \\
$?$ - incomparability & 0.5 & 0.5 \\
\hline
\end{tabular}


In this way, Table 3 is completely compatible with the use of the Usual preference function ([4]) in the deterministic case. This is particularly well suited for qualitative criteria. Imperfect information introduces the notion of incomparability as the local level as it becomes possible to hesitate as to which action should be preferred to the other when their evaluations are not known with certainty.

\section{NUMERICAL EXAMPLE}

Let's recall here the example used in [2] with four alternatives $a_{1}, a_{2}, a_{3}, a_{4}$ and four criteria of different nature: $f_{1}, f_{2}, f_{3}, f_{4}$ where $f_{1}$ is a stochastic criterion, $f_{2}$ is an evidential criterion, $f_{3}$ is a possibilistic criterion and $f_{4}$ is a fuzzy criterion. For each criterion, we have the corresponding performance matrix and the a priori information. The stochastic dominance indifference thresholds for all the criteria are $s_{1}=s_{2}=s_{3}=s_{4}=0.05$, and the weight distribution is: $\mathrm{Wj}=[0.4,0.2,0.3,0.1]$.

Table 4 gives the performance matrices for the four criteria. The evaluations according to $f_{4}$ are linguistic variables represented by trapezoidal fuzzy numbers $(a, b, c, d)$ (see figure 1).

Table 4. Performance matrices for $f_{1}, f_{2}, f_{3}, f_{4}$

\begin{tabular}{|c|ccccc|ccc|ccc|c|c|}
\hline$a_{i}$ & & & $\Omega_{1}$ & & & $\Omega_{2}$ & & $\Omega_{3}$ & $f_{4}$ & $\begin{array}{c}\text { Associated } \\
\text { fuzzy numbers }\end{array}$ \\
& $\omega_{1}^{1}$ & $\omega_{2}^{1}$ & $\omega_{3}^{1}$ & $\omega_{4}^{1}$ & $\omega_{5}^{1}$ & $\omega_{1}^{2}$ & $\omega_{2}^{2}$ & $\omega_{3}^{2}$ & $\omega_{1}^{3}$ & $\omega_{2}^{3}$ & $\omega_{3}^{3}$ & & \\
\hline$a_{1}$ & 3 & 1 & 2 & 6 & 4 & 90 & 90 & 110 & 35 & 70 & 45 & weak & $(0, .1,0, .2)$ \\
$a_{2}$ & 1 & 3 & 4 & 6 & 3 & 120 & 90 & 100 & 45 & 30 & 45 & fair & $(.5, .5, .2, .2)$ \\
$a_{3}$ & 2 & 1 & 2 & 3 & 5 & 110 & 130 & 80 & 45 & 60 & 25 & high & $(.9,1, .2,0)$ \\
$a_{4}$ & 6 & 1 & 4 & 4 & 2 & 80 & 110 & 120 & 40 & 70 & 30 & more or & $(.2, .2, .2, .2)$ \\
& & & & & & & & & & & & less weak & \\
\hline
\end{tabular}

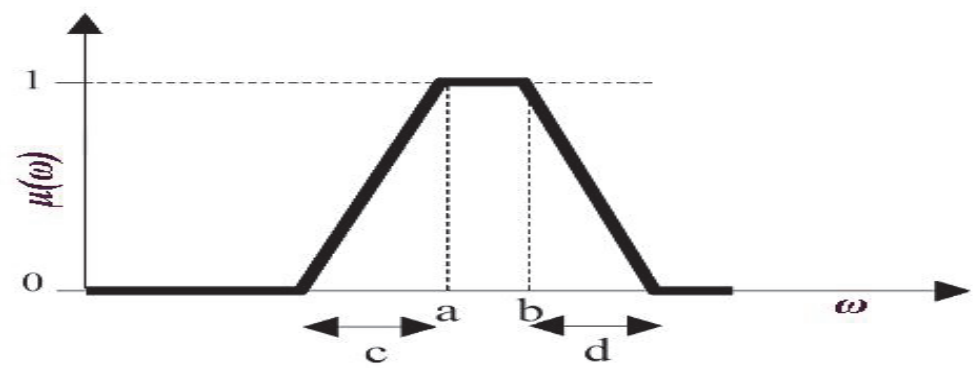

Figure 1. Membership function for fuzzy trapezoidal number $(a, b, c, d)$. 
Table 5 gives the a priori information for criterion $f_{1}$, while table 6 gives the a priori information for criteria $f_{2}$ and $f_{3}$. For criterion $f_{4}$, the a priori information is implicitly contained in the performance matrix.

Table 5. A priori information for $f_{1}$

\begin{tabular}{|c|ccccc|}
\hline & $\omega_{1}^{1}$ & $\omega_{2}^{1}$ & $\omega_{3}^{1}$ & $\omega_{4}^{1}$ & $\omega_{5}^{1}$ \\
\hline$P\left(\omega_{h}^{1}\right)$ & 0.28 & 0.23 & 0.10 & 0.28 & 0.11 \\
\hline
\end{tabular}

Table 6. A priori information for $f_{2}(j=2)$ and $f_{3}(j=3)$

\begin{tabular}{|c|c|c|c|c|c|c|c|c|}
\hline & $\varnothing$ & $\left\{\omega_{1}^{J}\right\}$ & $\left\{\omega_{2}^{J}\right\}$ & $\left\{\omega_{3}^{J}\right\}$ & $\left\{\omega_{1}^{J}, \omega_{2}^{J}\right\}$ & $\left\{\omega_{1}^{J}, \omega_{3}^{J}\right\}$ & $\left\{\omega_{2}^{J}, \omega_{3}^{J}\right\}$ & $\left\{\omega_{1}^{J}, \omega_{2}^{J}, \omega_{3}^{J}\right\}$ \\
\hline$m\left(B_{h^{\prime}}^{2}\right)$ & & & 0.1 & & 0.6 & & & 0.3 \\
\hline$m\left(B_{h^{\prime}}^{3}\right)$ & & & & 0.2 & & 0.2 & & 0.6 \\
\hline$\Pi\left(B_{h^{\prime}}^{3}\right)$ & 0.0 & 0.8 & 0.6 & 1.0 & 0.8 & 1 & 1 & 1 \\
\hline
\end{tabular}

Applying stochastic dominance results as in [2] has led to the local preference relations $H_{j}, j=1,2,3,4$ listed in Table 7 .

Table 7. Local preference relations $H_{j}, j=1,2,3,4$.

\begin{tabular}{|c|c|c|c|c|c|c|c|c|c|c|c|c|c|c|c|c|}
\hline & \multicolumn{4}{|c|}{$H_{1}$} & \multicolumn{4}{|c|}{$\mathrm{H}_{2}$} & \multicolumn{4}{|c|}{$\mathrm{H}_{3}$} & \multicolumn{4}{|c|}{$H_{4}$} \\
\hline & $a_{1}$ & $a_{2}$ & $a_{3}$ & $a_{4}$ & $a_{1}$ & $a_{2}$ & $a_{3}$ & $a_{4}$ & $a_{1}$ & $a_{2}$ & $a_{3}$ & $a_{4}$ & $a_{1}$ & $a_{2}$ & $a_{3}$ & $a_{4}$ \\
\hline$a_{1}$ & $*$ & $\approx$ & $\succ$ & $?$ & $*$ & $\succ^{-1}$ & $?$ & $?$ & $*$ & $\succ$ & $\succ$ & $\succ$ & $*$ & $\succ^{-1}$ & $\succ^{-1}$ & $\succ^{-1}$ \\
\hline$a_{2}$ & $\approx$ & * & $?$ & $\succ^{-1}$ & $\succ$ & $*$ & $?$ & $\succ$ & $\succ^{-1}$ & $*$ & $\succ$ & $\succ$ & $\succ$ & $*$ & $\succ^{-1}$ & $\succ$ \\
\hline$a_{3}$ & $\succ^{-1}$ & ? & $*$ & $\succ^{-1}$ & $?$ & $?$ & $*$ & $\succ$ & $\succ^{-1}$ & $\succ^{-1}$ & $*$ & $\succ^{-1}$ & $\succ$ & $\succ$ & $*$ & $\succ$ \\
\hline$a_{4}$ & $?$ & $\succ$ & $\succ$ & $*$ & $?$ & $\succ^{-1}$ & $\succ^{-1}$ & $*$ & $\succ^{-1}$ & $\succ^{-1}$ & $\succ$ & $*$ & $\succ$ & $\succ^{-1}$ & $\succ^{-1}$ & $*$ \\
\hline
\end{tabular}

Using the PROMETHEE preference coefficients suggested in Table 3, one can translate the obtained local preference relations $H_{j}, j=1,2,3,4$ into numerical values as shown in Table 8.

Multicriteria pair-wise preference index $\pi\left(a_{i}, a_{l}\right)$ are computed along with PROMETHEE leaving flow $\phi^{+}\left(a_{i}\right)$, entering flow $\phi^{-}\left(a_{i}\right)$ and net flow $\phi\left(a_{i}\right)$ as shown in Table 9. 
Table 8. PROMETHEE preference coefficients for the numerical example

\begin{tabular}{|c|cccc|cccc|cccc|cccc|}
\hline & \multicolumn{5}{|c|}{$H_{1}$} & \multicolumn{5}{|c|}{$H_{2}$} & \multicolumn{4}{c|}{$H_{3}$} & \multicolumn{4}{c|}{$H_{4}$} \\
\hline & $a_{1}$ & $a_{2}$ & $a_{3}$ & $a_{4}$ & $a_{1}$ & $a_{2}$ & $a_{3}$ & $a_{4}$ & $a_{1}$ & $a_{2}$ & $a_{3}$ & $a_{4}$ & $a_{1}$ & $a_{2}$ & $a_{3}$ & $a_{4}$ \\
\hline$a_{1}$ & $*$ & 0 & 1 & 0.5 & $*$ & 0 & 0.5 & 0.5 & $*$ & 1 & 1 & 1 & $*$ & 0 & 0 & 0 \\
$a_{2}$ & 0 & $*$ & 0.5 & 0 & 1 & $*$ & 0.5 & 1 & 0 & $*$ & 1 & 1 & 1 & $*$ & 0 & 1 \\
$a_{3}$ & 0 & 0.5 & $*$ & 0 & 0.5 & 0.5 & $*$ & 1 & 0 & 0 & $*$ & 0 & 1 & 1 & $*$ & 1 \\
$a_{4}$ & 0.5 & 1 & 1 & $*$ & 0.5 & 0 & 0 & $*$ & 0 & 0 & 1 & $*$ & 1 & 0 & 0 & $*$ \\
\hline
\end{tabular}

Table 9. PROMETHEE multicriteria indexes, leaving, entering and net flows

\begin{tabular}{|c|c|c|c|c|c|}
\hline Actions & $a_{1}$ & $a_{2}$ & $a_{3}$ & $a_{4}$ & $\phi^{+}\left(a_{i}\right)$ \\
\hline$a_{1}$ & $*$ & 0.3 & 0.8 & 0.6 & 1.7 \\
\hline$a_{2}$ & 0.3 & * & 0.6 & 0.6 & 1.5 \\
\hline$a_{3}$ & 0.2 & 0.4 & $*$ & 0.3 & 0.9 \\
\hline$a_{4}$ & 0.4 & 0.4 & 0.7 & $*$ & 1.5 \\
\hline$\phi^{-}\left(a_{i}\right)$ & 0.9 & 1.1 & 2.1 & 1.5 & \\
\hline$\phi\left(a_{i}\right)$ & 0.8 & 0.4 & -1.2 & 0 & \\
\hline
\end{tabular}

Depending on the decisional problematic, leaving, entering and net flows can be exploited according to PROMETHEE I or PROMETHEE II. In fact, using the net flow we obtain the PROMETHEE II complete ranking: $a_{1}, a_{2}, a_{3}, a_{4}$. In this case, the PROMETHEE I partial ranking induced by the leaving and entering flows is the same as the PROMETHEE II complete ranking.

Another approach to deal with imperfect information consists of replacing imperfect data by a punctual value that summarizes imperfect information. The use of expected values is one possible way of obtaining such summary values. When applied to the example at hand, this approach leads to the following PROMETHEE I partial ranking and PROMETHEE II complete ranking: $a_{1}, a_{2}, a_{3}, a_{4}$. One can see that this ranking is different from the one obtained above using the proposed approach. It is worth noticing the very different position of $a_{4}$ in both rankings. This can be explained by the larger uncertainty that affects the evaluations of $a_{4}$ on the most important criteria $f_{1}, f_{2}, f_{3}$. The proposed approach provides a lower, and thus, a more prudent ranking of $a_{4}$.

\section{Conclusion}

The proposed approach makes it possible to introduce jointly different modeling languages in order to take into account imperfection of information in the evaluation of the actions. It 
is thus possible to choose the best suited modeling language for each criterion in a same decision problem.

The approach has also several limits.

It corresponds to the use of the Usual preference function in the deterministic case and thus doesn't explicitly take into account the deviations between the evaluations in the pairwise comparisons. Future research will investigate how to integrate this aspect in the proposed approach.

The approach could also be extended to take into account imperfection of information in other multicriteria methods such as for instance the ELECTRE methods ([6]).

Imperfect information related to preference parameters (preference functions thresholds and criteria weights) is also an interesting topic that could be further investigated.

\section{ACKNOWLEDGMENT}

We would like to thank Prof. Wojtek Michalowski. Without him, this paper would probably have never been written.

\section{REFERENCES}

[1] M. Behzadian, R.B. Kazemzadh, A. Albadvi D. and M. Aghdasi, "PROMETHEE: A comprehensive literature review on methodologies and applications", European Journal of Operational Research, in press.

[2] S. Ben Amor, K. Jabeur and J-M. Martel, "Multiple criteria aggregation procedure for mixed evaluations", European Journal of Operational Research, 181(3), pp. 15061515, 2007.

[3] J.P. Brans and B. Mareschal "PROMCALC \& GAIA: A new decision support system for multicriteria decision aid”, Decision Support Systems, 12, pp.297-310, 1994.

[4] J.P. Brans and B. Mareschal "PROMETHEE Methods" in Multiple Criteria Decision Analysis: State of the Art, edited by J. Figueira, S. Greco and M. Ehrgott, pp.163-196, Kluwer, 2005.

[5] D. Dubois, H. Prade and S. Sandri, "On possibility/probability transformations", Lowen R, Roubens M (eds), Fuzzy Logic: State of the Art, Kluwer Academic Publ., Dordrecht, pp.103-112, 1993.

[6] J. Figueira, S. Greco and M. Ehrgott, Multiple Criteria Decision Analysis: State of the Art., Kluwer, 2005.

[7] A. Langewish and F. Choobineh, "Stochastic dominance tests for ranking alternatives under ambiguity", European Journal of Operational Research, 95, pp.139-154, 1996.

[8] B. Mareschal, "Stochastic multicriteria decision-making under uncertainty". European Journal of Operational Research 26 (1), 58-64, 1986.

[9] B. Mareschal and J.P. Brans "Geometrical representations for MCDA", European Journal of Operational Research, 39, pp. 284-292, 1989.

[10] J-M. Martel and K. Zaras, "Stochastic dominance in multicriterion analysis under risk", Theory and Decision, 39, 31-49, 1995.

[11]G. Munda, Multicriteria evaluation in a fuzzy environment, Physica-Verlag, Heidelberg, 1995. 
[12] B. Roy, Méthodologie Multicritère d'Aide à la Décision, Economica, Paris, 1985.

[13] G. Shafer, A Mathematical Theory of Evidence, Princeton University Press, Princeton N.J., 1976.

[14] Ph. Smets, "Decision making in the TBM: the necessity of the pignistic transformation", International Journal o Approximate Reasoning, 38, pp.133-147, 2005.

[15] $\mathrm{Ph}$. Smets, "Constructing the pignistic probability function in a context of uncertainty", in: Henrion M, Shachter RD, Kanal LN and Lemmer JF (eds), Uncertainty in Artificial Intelligence 5, North Holland, Amsterdam, pp.29-40, 1990.

Received July, 2011

\section{APPENDIX}

Table A.1. Performance matrix and expected values for $f_{1}$

\begin{tabular}{|c|cccccc|}
\hline & $\omega_{1}^{1}$ & $\omega_{2}^{1}$ & $\omega_{3}^{1}$ & $\omega_{4}^{1}$ & $\omega_{5}^{1}$ & $E\left(a_{i}^{1}\right)$ \\
\hline$a_{1}$ & 3 & 1 & 2 & 6 & 4 & 3.39 \\
$a_{2}$ & 1 & 3 & 4 & 6 & 3 & 3.38 \\
$a_{3}$ & 2 & 1 & 2 & 3 & 5 & 2.38 \\
$a_{4}$ & 6 & 1 & 4 & 4 & 2 & 3.65 \\
\hline$P\left(\omega_{h}^{1}\right)$ & 0.28 & 0.23 & 0.10 & 0.28 & 0.11 & \\
\hline
\end{tabular}

Table A.2. Performance matrix, pignistic probabilities and expected values for $f_{2}$

\begin{tabular}{|c|cccc|}
\hline & $\omega_{1}^{2}$ & $\omega_{2}^{2}$ & $\omega_{3}^{2}$ & $E\left(a_{i}^{2}\right)$ \\
\hline$a_{1}$ & 90 & 90 & 110 & 92 \\
$a_{2}$ & 120 & 90 & 100 & 103 \\
$a_{3}$ & 110 & 130 & 80 & 117 \\
$a_{4}$ & 80 & 110 & 120 & 99 \\
\hline $\operatorname{Bet} P\left(\omega_{h}^{2}\right)$ & 0.4 & 0.5 & 0.1 & \\
\hline
\end{tabular}


Table A.3. Performance matrix, pignistic probabilities and expected values for $f_{3}$

\begin{tabular}{|c|cccc|}
\hline & $\omega_{1}^{3}$ & $\omega_{2}^{3}$ & $\omega_{3}^{3}$ & $E\left(a_{i}^{3}\right)$ \\
\hline$a_{1}$ & 35 & 70 & 45 & 47 \\
$a_{2}$ & 45 & 30 & 45 & 42 \\
$a_{3}$ & 45 & 60 & 25 & 38 \\
$a_{4}$ & 40 & 70 & 30 & 41 \\
\hline $\operatorname{BetP}\left(\omega_{h}^{2}\right)$ & 0.3 & 0.2 & 0.5 & \\
\hline
\end{tabular}

Table A.4. Performance matrix and expected values for $f_{4}$

\begin{tabular}{|c|cccc|c|}
\hline & $a$ & $b$ & $c$ & $d$ & $E(a, b, c . d)$ \\
\hline$a_{1}$ & 0 & 0.1 & 0 & 0.2 & 0.10 \\
$a_{2}$ & 0.5 & 0.5 & 0.2 & 0.2 & 0.50 \\
$a_{3}$ & 0.9 & 1 & 0.2 & 0 & 0.90 \\
$a_{4}$ & 0.2 & 0.2 & 0.2 & 0.2 & 0.20 \\
\hline
\end{tabular}

Table A.5. Performance matrix using expected values $E\left(a_{i}^{j}\right)$

\begin{tabular}{|l|cccc|}
\hline & $f_{1}$ & $f_{2}$ & $f_{3}$ & $f_{4}$ \\
\hline$a_{1}$ & 3.39 & 92 & 47 & 0.1 \\
$a_{2}$ & 3.38 & 103 & 42 & 0.5 \\
$a_{3}$ & 2.38 & 117 & 38 & 0.9 \\
$a_{4}$ & 3.65 & 99 & 41 & 0.2 \\
\hline
\end{tabular}

